\title{
Drives and Motion Control Teaching based on Distance Laboratory and Remote Experiments
}

\author{
Markus A. Vogelsberger*, Peter Macheiner*, Pavol Bauer**, and Thomas M. Wolbank ${ }^{\dagger}$ \\ ${ }^{* *}$ Institute of Electrical Drives and Machines, Vienna University of Technology, Vienna, Austria \\ ** Delft University of Technology, Delft, The Netherlands
}

\begin{abstract}
This paper presents the organisation and the technical structure of a remote controlled laboratory in the field of high dynamic drives and motion control. It is part of the PEMCWebLab project with the goal of providing students with practical experience on real systems in the field of power electronics and drives. The whole project is based on clear targets and leading ideas. A set of experiments can be remotely performed on a real system to stepwise identify a two axes positioning system and to design different cascaded control loops. Each single experiment is defined by its goals, the content of how to achieve them, and a verification of the results as well as the achieved learning outcomes. After a short description of the PEMCWebLab project, the structure of the remote control is presented together with the hardware applied. One important point is error handling as real machines and power electronics are applied. Finally, a selection of experiments is presented to show the graphical user interface and the sequence of the laboratory.
\end{abstract}

Key Words: Drives, Education, Motion control, Power electronics, Remote Laboratory

\section{INTRODUCTION}

Although modelling and dynamic simulation are basic tools for understanding and verifying theoretical subjects, experimentation with a real system plays a fundamental role that cannot be replaced. An analysis of these tools from the point of view of modern education is given in [1], [2]. Practical education needs to be based on understanding the phenomena that occur in real systems. Remote control of experiments through the internet comes as a solution to these problems, allowing students to control them, without leaving their normal workplace.

The rapid development of information and communication technology (ICT) beginning in the 90s enabled the expansion of online distance laboratories. Their utilisation presents the latest trend in education - to get practical experience by experimentation with measurements, verifying properties of complex equipment as well as an analysis of the equipment in various operation points. By taking advantage of the internet and the developments in related technologies, an increasing number of remote access solutions are being developed. Remote hardware experiments are adapted in such a way that they can be accessed from the web, which enables distance sharing of the experiment by various individuals and/or institutions.

\footnotetext{
Manuscript received Mar. 3, 2010; revised Jul. 14, 2010

$\dagger$ Corresponding Author: thomas.wolbank@tuwien.ac.at

Tel: +43-1-58801-37226, Fax: +43-1-58801-937226, Vienna Univ. of Tech.

* Institute of Electrical Drives and Machines, Vienna University of Technology, Austria

** Delft University of Technology, The Netherlands
}

Currently we can find numerous solutions for remote controlled experiments in various fields of practical education and also there are numerous projects running whose developments have been presented at special conferences, e.g. in [3], [4] and at special sessions of scientific conferences [1], [8], [9], [11]-[14], etc.

An overview of some typical solutions is presented in [1]. Within the PEMCWebLab a more complex system of distance and virtual laboratories was developed containing not only single experiments but a whole set of experiments [2] that are distributed across Europe. The distance and virtual laboratories are not web-based simulations. They present real electrotechnical experiments conducted in the laboratory. However, they are remotely controlled and monitored by web-based tools with the visualization of measuring apparatus, electronic components and many other factors. The experiments cover the basic fields of electrical engineering. They are placed in different laboratories in universities. The developed system presents an open system enabling later expansion.

\section{PEMCWEBLAB.COM SYSTEM}

To support distance learning in electrical engineering a set of remotely controlled real experiments from fields of electrical engineering, mainly from industrial electronics (power electronics, electrical drives and motion control), has been developed within the Leonardo da Vinci EU supported project, creating the PEMCWebLab. It can be approached via the webpage www.PEMCWebLab.com, where there is also a direct link to the booking system and more information about the 
project, dissemination results and a description of the different modules (measurements).

The PEMCWebLab creates an integrated learning platform. Several learning issues are addressed such as:

- Learning objectives

- Education

- Animation

- Simulation

- Experimentation

In the first part, the learning objectives of each experiment are addressed. In the education part, the theoretical background of each individual experiment is given. Interactive animations developed in previous projects are addressed too. The last educational method before experimentation is simulation.

\section{A. Learning objectives}

The experiments are not only analysis oriented (to measure and see the results) but also synthesis oriented. They should involve a design aspect. Therefore the measurements are designed as a project with an educational philosophy.

The learning objectives for each single experiment are defined with the following goals:

- the objectives should be described in terms of knowledge and/or skills to be acquired by students,

- the objectives should be realistic given the attributes of the target group (prior knowledge, skills) and the time available for the students (credit points),

- the outcomes should be testable; if not, they should be left out or reformulated,

- it should be possible to analyze the functions and workings of electrical power (electronic) systems,

- it should be possible to design, implement and verify electrical power (electronic) systems.

Since both analysis and synthesis are key competences of engineers, the main objectives of this project are highly appropriate in an engineering education environment. A few remarks should be made with respect to these issues. Analyzing existing systems implies measurements. It is, however, very important that students learn to conceive measurements as a means, rather than a goal. Measurements are a means to verify a hypothesis on the functioning of a system under analysis. The modules should indeed be structured such, that this concept becomes clear. Therefore, it is proposed that any measurements be embedded in the following structure of consecutive steps:

- Define the function of the system

- Decompose into subsystems with sub-functions

- Formulate hypotheses on the characteristics of the subsystems

- Design measurements for verification of the hypotheses

- Carry out the measurements

- Compare the results with the hypotheses

- Evaluate learning

As far as synthesis is concerned, all modules will lead to a common design methodological approach for students.

The main function of the PEMCWebLab is to provide a web-based remote control system for designed experiments.
The learning process includes several, specially designed, experimental tasks.

After completion of the online experiments, the learners are given an opportunity to take a simple questionnaire or alternatively to submit their report through the available feedback subsystem for its final evaluation (depending on the requirement of the instructor).

An example of an experiment from the PEMCWebLab is given. This experiment is used for the motion control course. First a description of the system is given and then three assignments namely machine parameter identification, calibration of the resolver, and current control loop design are shown in detail with the goals of the experiment, a detailed explanation with the results and a verification of the achieved learning outcomes.

\section{DESCRIPTION OF THE SYSTEM}

\section{A. Mechanical system}

During the laboratory course "motion control" students will have the opportunity to perform tests and experiments on the topics of field oriented control, inverter fed operation, and cascaded multilevel control. The mechanical system operated is a two axes positioning system fed by permanent magnet synchronous machines (PMSM).

The whole arrangement of the mechanical system can be seen in Fig. 1. It consists of two linear axes $(6,7)$ each equipped with a lead screw that is driven by a permanent magnet synchronous machine (PMSM) $(4,5)$. The second axis (y-axis, 7) is mounted on top of the first (x-axis, 6). Each of the PMSMs is fed by an individual inverter (1) and control electronics $(2,3)$ thus allowing separated movement of the two carriages. On the carriage of the second axis (y-axis, 7) a lifting magnet is fixed allowing a third axis movement (pen up/down). The maximum working range of the two axes is $300 \mathrm{~mm}$.

\section{B. Power electronics - inverter}

The power electronics is realized by two voltage source inverters fed on the main side by $3 \times 400 \mathrm{~V}$ with a diode bridge. The nominal dc link voltage is thus $560 \mathrm{~V}$ dc. The inverter current (dc link plus output; 5A max.) and the dc link voltage ( $600 \mathrm{~V}$ max.) are monitored and connected to an emergency shutdown circuit. All security critical signals are processed by hardware as well as by software. A chopper circuit protects the dc link from overvoltage during regenerative operation.

\section{System security}

As the system and control parameters can be changed by students, possible unstable operating conditions have to be considered. To avoid damage or destruction of the whole arrangement a set of security measures are realized at different levels.

The first and uppermost security check is made in the user interface. All parameters that can be changed by the user are checked to stay within a tolerable limit for non-destructive operation. 


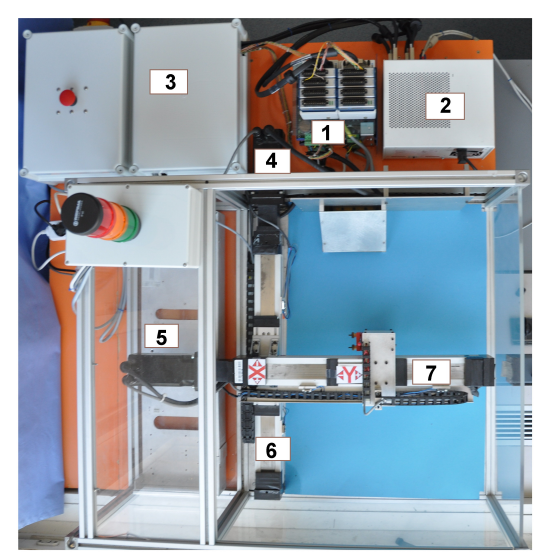

Fig. 1. Mechanical arrangement of the two axes positioning system.

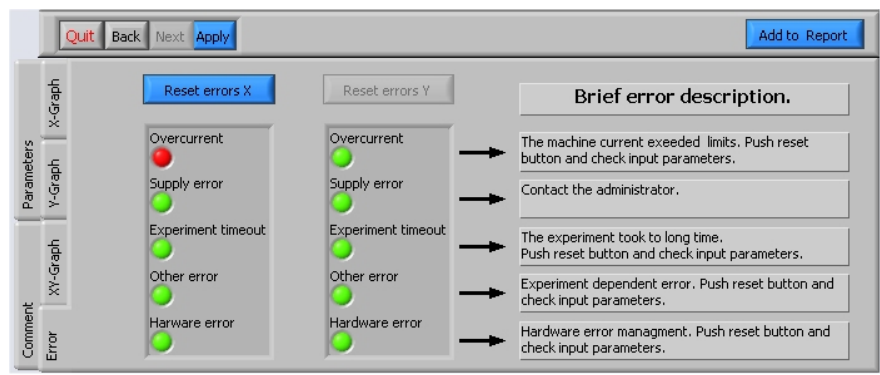

Fig. 2. Error tab in graphical user interface.

A second security measure is the duration of each experiment as well as the reference input values. All of the reference values for system identification are predefined within a specific range. In addition the user can choose from different time traces for the reference functions. To avoid mechanical resonances each experiment is automatically terminated after a defined duration. This duration is chosen to be long enough to deliver enough results for a grading of the system performance and short enough to avoid damage by vibration or instability.

The next security check is done by software during the experiment. All critical system parameters and especially the inverter currents and the mechanical position are monitored. In case of an over current warning the inverter is disabled. If a position warning is active an emergency software routine is activated that tries to generate a braking torque to stop the system before the end position of the corresponding axis is reached.

The lowest security level is done exclusively by hardware. If an over current, temperature, or voltage is detected by a sensor the inverter is disabled immediately. The same is done if a critical mechanical position is detected by one of the endposition switches. The final end position is protected by elastic catch stops.

A system that is too robust to be overturned would however, be too much like a simulation. It is thus necessary to give students the feeling that a false experiment setup or the wrong parameters have consequences that may shut down the whole system. Therefore a special error handling structure was included to give students a possible negative feedback on their actions.
When a system error is detected the "error tab" will be laid on top of the user interface with the corresponding indicator marked red. The same tab also gives a short error description. If, for example an over current error in the $\mathrm{x}$-axis occurred (Fig. 2), the student should check the "X-Graph" tab to see the current values sampled during the experiment. This error is generated by hardware when the current clearly exceeds the rated value. Usually the voltage applied was to high.

The threshold levels for these user errors are chosen on the save side to avoid the activation of critical system errors.

\section{Control electronics}

The control electronics of the whole system are realized on an industrial FPGA using a high level programming language (Labview). To avoid time consuming debugging and repetitive compiling, all of the software loops necessary for carrying out experiments are predefined. Students can thus focus on the measurements, the control structure, the identification of the system parameters, and the performance of different control loops. Each axis of the positioning system is controlled by an individual FPGA. The two systems are linked together by a hardware input/output connection to enable synchronous operation. Both of the FPGA systems communicate with a real-time system. An experiment identifier together with the parameters of the current experiment are send from the realtime system to the corresponding FPGA to start each experiment. During the experiment the measured data are streamed from the FPGA to the real-time system. The communication of the real-time system and the user interface is done via TCPIP. The actual user interface and the web-page is hosted on a PC. There all of the data is collected and finally displayed on a web-page where the stream of a web-cam is included. This PC holds a connection to the internet.

This clear physical separation of the systems ensures a high security level for the whole system. Even a system crash in the operating system of the web-connected PC will not influence the running experiment or the different hardware security levels.

\section{E. Electric machines}

Each machine driving an axis is a synchronous machine with permanent magnets (PMSM). The machine data is as follows:

Nominal Voltage: $U_{N, \text { eff }}=560 \mathrm{~V}$

Nominal Current: $I_{N, e f f}=2,4 \mathrm{~A}$

Nominal torque: $M_{N}=1,72 \mathrm{Nm}$

Nominal speed: $n_{N}=7200 \mathrm{~min}-1$

Number of pole pairs: $p=3$

\section{F. Web interface}

After booking the laboratory for a specific time slot the student is able to access the web page via a dynamic link. The web page (Fig. 3) contains a graphical user interface (GUI) (top left) where at first a login has to be performed. In the lower part of the GUI the experiments are controlled and the measurement results are displayed. In the lower part of the 


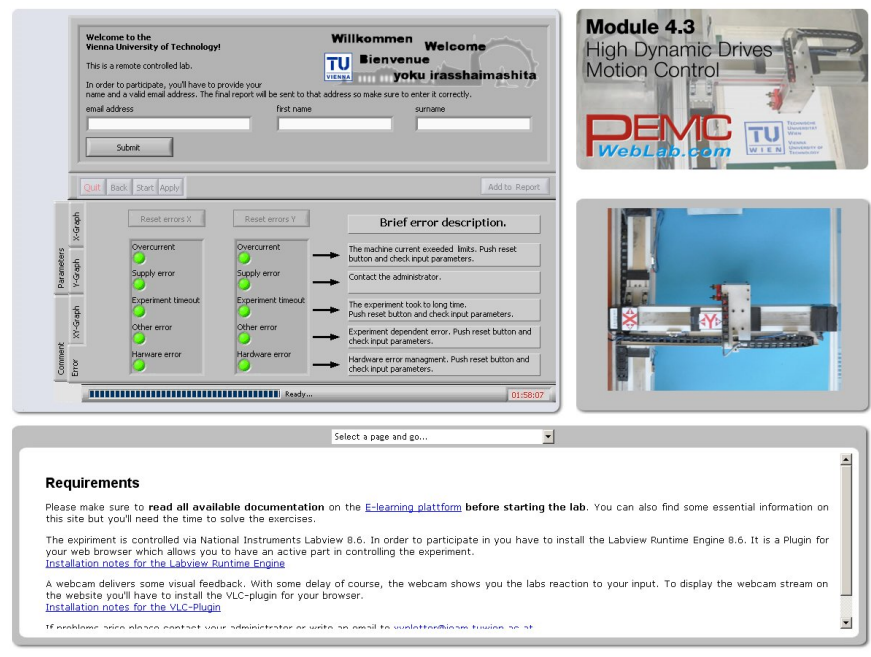

Fig. 3. Login screen and webpage of the laboratory.

web page a short description of each experiment is given. The video stream of the webcam is displayed in the lower right part of the GUI to give feedback on the movement of the two axes during the experiments.

Depending on the speed of the internet connection there is a time delay in the video displayed of up to several seconds with respect to the measurement results.

\section{SYSTEM IDENTIFICATION AND PARAMETER TUNING (DESCRIPTION OF THE EXPERIMENTS)}

In the following section the experiments are described to stepwise identify the necessary system parameters for complete control of the system. When necessary some theoretical explanations are given.

\section{A. Machine parameter identification}

Goal of the experiment:

Identification of the machine parameters, like the stator resistance, stator inductance and the stator time constant, is absolutely necessary for any executable drive operation.

The identification of the time constant is done during the first experiment by establishing a simple voltage step to the machine using the inverter and by exploiting the current response. The time duration of the current needs to rise from zero to $63.2 \%(5 / 8)$ of its final steady state value will be identified and labeled as the stator time constant $\left(t_{S}\right)$.

In the second part of this experiment an identification of the stator resistance will be realized. Due to the disturbing impact of the inverter dead time, two measurements are necessary and must be combined. Hence the stator resistance can be identified and calculated using the current and voltage values from two different adjusted voltage phasors.

To start an experiment, a student has to type into the GUI the amplitude of the normalized stator voltage space phasor he or she wants to apply. After pressing the blue button marked "apply" (Fig. 4) the experiment starts. When the experiment is finished, the "X-Graph" tab is moved to the top of the screen and the measured time trace of the stator current is displayed.

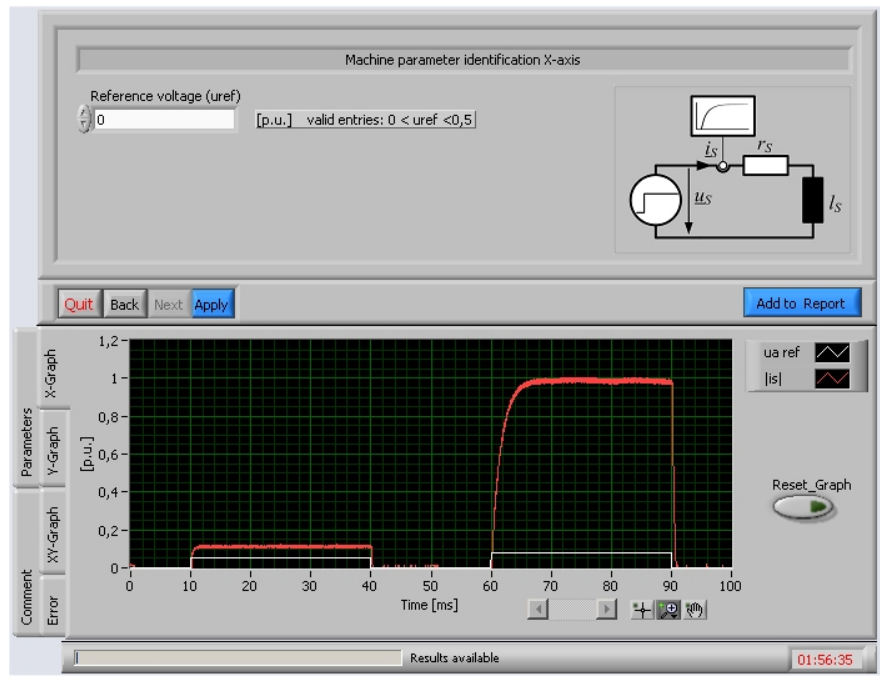

Fig. 4. Screenshot of time plot for experiment "machine parameter identification".

The student may use the buttons on the lower right side of the graph to zoom the display and to determine the exact time constant and the resulting current values.

Once the parameters have been identified by the student he or she may proceed to the next step. Pressing the blue button marked "Add to Report" will store the measurement data of the X-Graph and then change the "Next" button to blue to indicate that the student may go on. In the following input mask the identified parameters are entered and stored to be used for all further experiments.

Verification of the achieved learning outcomes:

The identified machine parameters are checked by the system. If their values stay within a tolerable range the student may go on to the next experiment. If not the current experiment has to be repeated. The wrong identified parameters may lead to poor performance of the drive or even to the generation of a user error in one of the following experiments.

\section{B. Calibration of the resolver}

Goal of the experiment:

As mentioned above the rotor position is determined using a resolver. Though the resolver delivers an absolute position signal it has to be calibrated with respect to the axis of the magnets (d-axis) as the mounting of the resolver on the rotor shaft is arbitrary and therefore unknown.

This calibration is done during the next experiment. The idea of this experiment is to establish a current phasor in the machine windings that is strong enough to produce a torque that is sufficiently higher than the friction torque. As a result the rotor of the PMSM will rotate towards the direction of the current phasor. After some oscillations the d axis (direction of the permanent magnet flux phasor) will be aligned with the axis of the current phasor. The angle of the current phasor can be set to any value between $+/-1$ corresponding to $+/-\pi$ in the GUI (Fig. 5). Its default value is zero, corresponding to the direction of the real axis. There are no parameters to be adjusted before this calibration. The experiment is started 


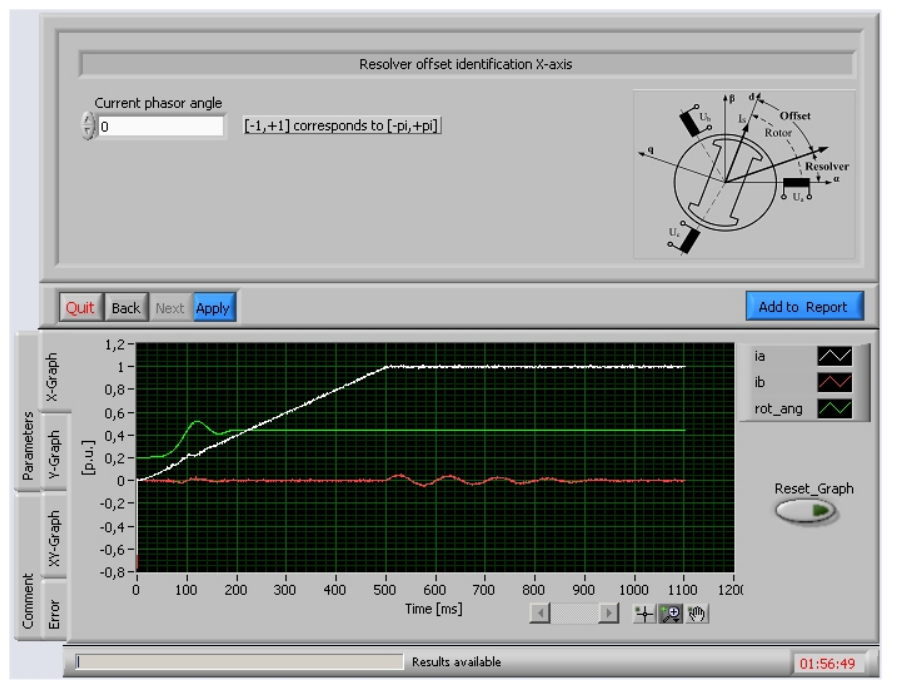

Fig. 5. Screenshot of graphic user interface for experiment "calibration of resolver".

by clicking on the "Apply" button. It is automatically stopped after the corresponding time and the results are displayed.

The result is shown in Fig. 5 for the $\mathrm{x}$-axis drive. The green trace displayed in the figure shows the time course of the resolver position signal scaled in p.u. on the vertical axis. The oscillations of the rotor as well as the end position, marking the resolver signal with the aligned d-axis, are clearly visible. As the current phasor angle is zero the resolver end position (with an aligned d-axis) should also deliver zero degrees. The end value of the resolver thus directly delivers the resolver offset position.

The remaining traces (white and red) show the stator current components $(\alpha$ and $\beta$ ) in the stator fixed reference frame. As the angle of the current phasor was chosen to be zero in the GUI, the real component $(\alpha)$ "ia" corresponds to the current magnitude (white). This magnitude is increased slowly during the experiment to avoid excessive vibration of the system. The student may zoom to specific areas of the display using the buttons on the lower right area of the diagram.

Note: As the machines pole pair number is three there are three possible results for the resolver offsets each $120^{\circ}$ apart. If the experiment is repeated, the rotor is turned by the system to a different (arbitrary) position at least $+/-120^{\circ}$ away to avoid starting the experiment in an 'aligned' rotor position and to ensure that the other possible results are presented. Pressing "Add to Report" stores the measured data, displays an input mask, and turns the "Next" button blue.

The identified result of this experiment has to be entered and then it is automatically saved when the "Next" button is clicked. It is then used for all further experiments throughout the whole laboratory course.

Verification of the achieved learning outcomes:

The identified resolver offset has to be typed in by the student. If the values stay within a tolerable range the student may proceed to the next experiment. If not the current experiment has to be repeated. A poorly calibrated resolver will lead to reduced performance of the drive or even to the generation of a system error in the following experiments.
At this stage the student has learned to identify the orientation of the magnets on the rotor (d-axis directions) of a six pole PMSM. This means, the angle information of the three d-directions along the circumference of the machine, each 120 (mechanical) apart, was identified by establishing a single current space phasor to the machine.

In addition, the difference between the mechanical and the electrical angle information and the interrelation by the number of pole pairs are also understood.

\section{Current control loop design}

Goal of the experiment:

Identification of the system parameters is necessary to tune the current control loop. As the machine is driven by a voltage source inverter, the output of the current controller is a reference voltage. In order to calculate the optimum voltage necessary to accurately adjust the reference current, the correlation between the voltage and the current has to be identified.

The control loop structure for both axes of the system is the same. The system description is written independent of the axis. However, as the system parameters for the two axes are different, the same experiments/calculations have to be done for each axis separately.

The current control is the innermost control loop of the drive software. Its input value is the reference current phasor and the output is the reference voltage that is further processed in the PWM and the inverter.

Looking at the stator equation of the PMSM (1) it can be seen that the back emf (time derivative of the stator flux linkage $\underline{\Psi}_{S}$ ) acts as a disturbance for the current control loop.

The loop is realized in the rotor fixed $\mathrm{d}-\mathrm{q}$ reference frame to enable constant reference values for the constant torque independent of the speed.

$$
\underline{u}_{S}=\underline{i}_{S} \cdot r_{S}+\frac{d \underline{\Psi}_{S}}{d \tau}+j \omega_{K} \cdot \underline{\Psi}_{S} .
$$

Considering the flux linkage equation and the constant flux linkage $\left(\Psi_{M}\right)$ of the permanent magnets $\Psi_{S}=l_{S} \cdot i_{S}+\Psi_{M}$ as well as a rotor fixed reference frame leads to:

$$
\underline{u}_{S}=\underline{i}_{S} \cdot r_{S}+l_{S} \frac{d \underline{i}_{S}}{d \tau}+j \omega_{m} \cdot \underline{\Psi}_{M}
$$

with $\omega_{m}$ as the rotor speed. The term $j \omega_{m} \Psi_{M}$ (back emf) acts as a disturbance in the current control.

In the control loop this disturbance has to be considered as well as the fact that the inverter has some non-ideal properties like dead time.

The overall structure of the control loop is as follows:

Replacing the different blocks in the loop by the transfer functions represented in the frequency-domain after applying the Laplace transform leads to Fig. 7.

The parameters of the loop are: the proportional gain of the PI controller $\left(K_{C C}\right)$, the integral part of the PI controller $\left(\tau_{C C}\right)$, the dead time of the inverter $\left(\tau_{c o n v}\right)$, the dead time of the current measurement $\left(\tau_{M}\right)$, the stator resistance $\left(r_{S}\right)$, and the stator inductance $\left(l_{S}\right)$. 


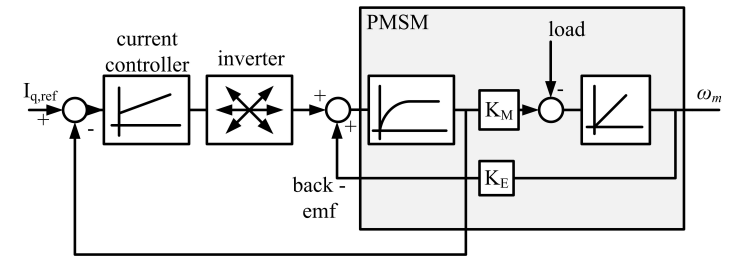

Fig. 6. Structure of the system showing the current control loop.

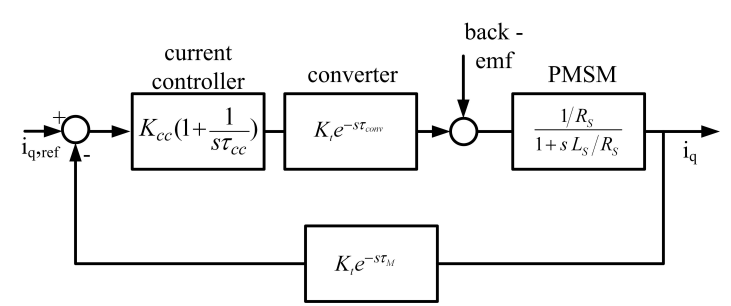

Fig. 7. Mathematical abstraction of the current control loop.

In the control unit all values are represented in the per unit (p.u.) form. Thus the time values $(t)$ are also scaled to the p.u. form $(\tau)$ according to the nominal angular velocity of the machine (III.E. index $_{N}$ )

$$
\begin{aligned}
& \omega_{\text {scale }}=2 \cdot \pi \cdot n_{N} \cdot p \\
& T_{\text {scale }}=1 / \omega_{\text {scale }}=0,442 \mathrm{~ms} \quad \tau=\frac{t}{T_{\text {scale }}} .
\end{aligned}
$$

The values of the voltages, currents, resistance, and inductance are also scaled to p.u. (using the peak values of the nominal phase current and the phase voltage as scaling factors).

For the control circuit, the time duration of the whole signal path from the output of the current controller to the terminals of the machine are all contained in the value "inverter dead time". The time delay of the measurement gives the calculation time of the control electronics between the sampling of the current sensors till the new output value of the current controller is adjusted (index ${ }_{C M}$ ).

For the design of the current controller the disturbing influence of the back emf is neglected as well as the crosscoupling between the two phasor components (this will be considered in a later experiment).

The transfer function of the plant (inverter + machine + measurement) can be represented by the following equation containing two dominant time constants. The small time constants of the system (the inverter-dead-time and the measurement) are both combined to $T_{2}$ whereas the constant $T_{1}$ is determined by the machine parameters $l_{S}$ and $r_{S}$.

With the dead time of the inverter being $T_{\text {conv }}=3,0 \mu \mathrm{s}$ and the dead time of the current measurement (control loop) as $T_{C M}=200 \mu s$ the following is obtained:

$$
\begin{aligned}
& \tau_{\text {Conv }}=\frac{T_{\text {Conf }} / 2}{T_{\text {scale }}}=3,39 \cdot 10^{-3} \\
& \tau_{M}=\frac{T_{C M}}{T_{\text {scale }}}=0,452 .
\end{aligned}
$$

The inverter dead time effective for the control loop is

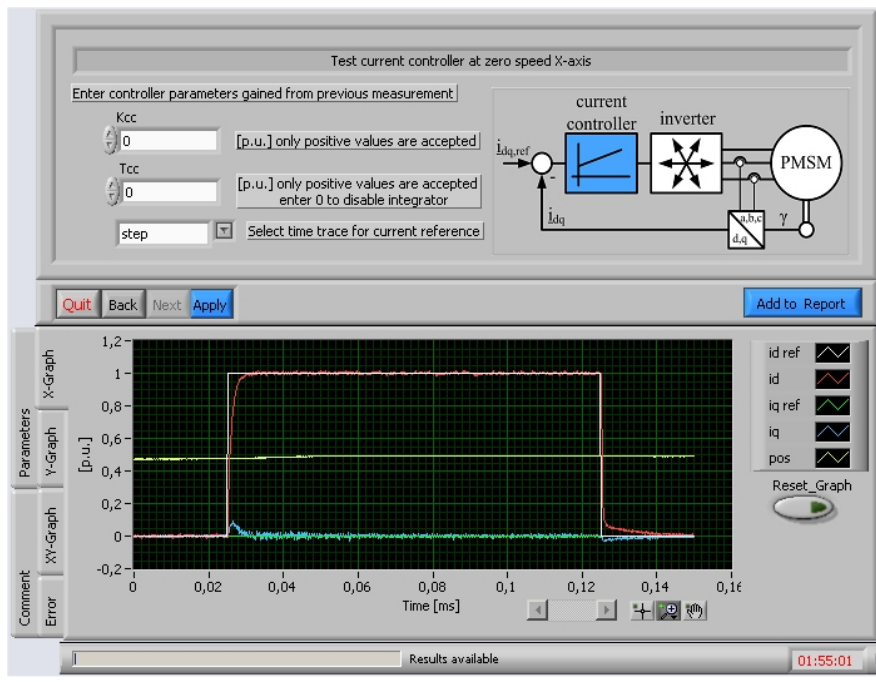

Fig. 8. Screenshot of graphic user interface and results window for experiment "current control loop design".

approximated by half the value adjusted for the inverter which leads to:

$$
\tau_{2}=\tau_{\text {Conf }}+\tau_{M}=0,455 .
$$

The equation of the plant can thus be written as:

$$
G(s)=\frac{\frac{1}{R_{S}}}{\left(1+s \frac{L_{S}}{R_{S}}\right) \cdot\left(1+s T_{s}\right)}=\frac{K_{s}}{\left(1+s T_{1}\right) \cdot\left(1+s T_{2}\right)} .
$$

The equation of the PI current controller $\left(\right.$ index $_{C C}$ ) is:

$$
K(s)=K_{c c}\left(1+\frac{1}{s \tau_{c c}}\right) .
$$

The value of the time constant $\tau_{1}$ can be determined using the machine parameters $r_{S}$ and $l_{S}$ as follows:

$$
\tau_{c c}=\tau_{1}=\frac{l_{S}}{r_{S}} .
$$

The current controller will be designed according to the optimum magnitude method. This means that the transfer function of the closed control loop should have a constant magnitude of 1 along an - as high as possible - frequency range. To achieve this as many derivatives of the equation as possible have to be zero. This leads to the following design criteria for $K_{C C}$ (for details see control literature):

$$
K_{c c}=\frac{\tau_{1}}{2 \cdot \tau_{2} \cdot K_{s}}=\frac{\frac{l_{S}}{r_{S}}}{2 \cdot \tau_{2} \cdot \frac{1}{r_{S}}}=\frac{\tau_{1} \cdot r_{S}}{2 \cdot \tau_{2}} .
$$

These control parameters can now be set in the GUI either by typing in the values or by clicking the arrow buttons. The value of $K_{C C}$ corresponds to the p-factor in the GUI and the value of $\tau_{C C}$ corresponds to the $\mathrm{i}$-factor.

The performance of the current control loop is tested by the next experiment, which can be started after entering the control parameters and clicking the "Apply" button. In 
this experiment the student may choose between the step, rectangular, or sinusoidal reference functions to be used as the current reference value. To avoid movement of the rotor, the reference value is always applied in direction of the flux (d-axis).

After the experiment is stopped the measurement results are displayed in the lower part of the GUI. The scaling of the vertical axis is done in p.u. values.

The white trace gives the reference (step) function of the d-current ("id_ref"). The measured current is displayed red ("id"). Again it is possible to zoom in using the buttons on the lower left side of the diagram. The q-current component ("iq") should stay around zero according to its reference value ("iq_ref") and the rotor position ("pos") should remain constant during the experiment.

Students may change the calculated values to compare the control performance for different parameters. Once the performance is considered "optimum" the corresponding result and parameters may be stored in the system and added to the report by clicking the appropriate button.

Note: For the calculation of the control parameters some assumptions have been made for simplification. Effects not considered like sensor and measurement noise, the nonlinearity of the inverter/power electronics, and the dependence of the inductance level on the spatial direction $(\mathrm{d}, \mathrm{q})$ lead to a slightly different (non-ideal) system behaviour. As a result it is possible to manually tune the control parameters by starting from the calculated values. Thus the system performance may be slightly increased.

Verification of the achieved learning outcomes:

The chosen parameter values have to be entered by the student. These values are checked to stay within a tolerable range. In addition, the control performance is verified by processing the measurement results of the current deviation into some sort of quality function. If the control performance is tolerable the student may proceed to the next experiment. If not the experiment has to be repeated.

Concluding remark:

After modelling the real system (controller and plant) to their equivalent mathematical transfer functions, the normalized plant time constants are build.

A proportional-integral PI-controller was selected as a current controller, by using the optimum magnitude method as a design strategy. After parameter identification, the whole current control loop was tested though experiments to optimize the control parameters.

The student has learned how to identify the system parameters and how to design the current controller. Now the innermost control loop of the whole system is well turned.

\section{CONCLUSIONS}

In this paper the PEMCWebLab project for distance and virtual laboratories is introduced and illustrated with three experiments from the remote laboratory in the area of motion control as an example. Each single experiment has concrete learning objectives, that are evaluated as part of the experiment.
Although it is too soon to evaluate the overall efficiency of the mentioned educational approach the first experience is more then encouraging.

\section{ACKNOWLEDGMENT}

This work has been performed within the E-learning Distance Interactive Practical Education (EDIPE) project (project No CZ/06/B/F/PP-168022). It was supported by the EC within the framework of the Leonardo da Vinci II programme. The opinions expressed by the authors of this paper do not necessarily reflect the position of the EC, nor does it involve any responsibility on its part.

\section{REFERENCES}

[1] P. Bauer, V. Fedak, V. Hajek, and I. Lampropoulos, "Survey of distance laboratories in oower electronics," Proceedings of IEEE 39th Annual Power Electronics Specialists Conference, pp.430-436, 2008.

[2] E-learning Distance Interactive Practical Education - EDIPE, Pilo Project of the EU Leonardo da Vinci Vocational Training Programme. Project No CZ/06/B/F/PP-168022, duration 10/2006 - 09/2008, at http://www.powerweblab.com/.

[3] F. Coito, L. Gomes, and A. Costa, "Simulation, Emulation and Remote Experiments," Proceedings of the Workshop on using VR in Education, pp. 99-110, Mar. 2007.

[4] Proceedings of International Conference on Remote Engineering and Virtual Instrumentation (REV'07), www.i-joe.org/ojs/, Jun. 2007.

[5] F. G. Lerro and M. D. Protano, "Web-based remote Semiconductors Devices Testing Laboratory," International Journal of Online Engineering (iJOE), pp. 161-164, 2007.

[6] K. Yeung and Huan, J., "Development of a Remote-Access Laboratory: a DC Motor Control Experiment," Computers in Industry, Vol. 52, Issue 3, pp. 305-311. Dec. 2003.

[7] V. Silva, V. Carvalho, R. M. Vasconcelos., and F. Soares, "Remote PID control of a DC motor," International Journal of Online Engineering (iJOE), p. 147, 2007.

[8] D. Maga, J. Sitar, and J. Dudak, "Measurement of electrical machines in virtual laboratory," 10th Int. Symposium on Mechatronics "Mechatronika 2007, Jun. 2007.

[9] E. Ernest; R. Sztylka; B. Ufnalski; and W. Koczara, "Methods in teaching modern ac drives: inverter-fed motor system with internet-based remote control panel," 12th International Power Electronics and Motion Control Conference, pp. 2130 - 2133, 2006.

[10] A. Rojko, D. Hercog, and K. Jezernik, "Advanced control course with teleoperation in the mechatronics study," 16th Int. Conf. on Electrical Drives and Power Electronics, Sep. 2007.

[11] V. Fed?k, T. Balogh, P. Bauer, and S. Jusko, "Virtual and Remote Experimentation in Motion Control," Int. Conference on Mechatronics, May 2008.

[12] P.Bauer, J.Dudak, D.Maga, V.Hajek, "Distance practical education for Power Electronics," International Journal of Engineering Education (IJEE), Vol.23, No.6, 2007.

[13] D.Sobczuk: "Internet Based Teaching of Pulse Width Modulation for Three-Level Converters," In Proceedings of EUROCON 2007, Sep. 2007.

[14] D.Sobczuk, "E-Learning Presentation Of Pulse Width Modulation Methods for Two And Three-Level Converters," International conference EDPE, Sep. 2007.

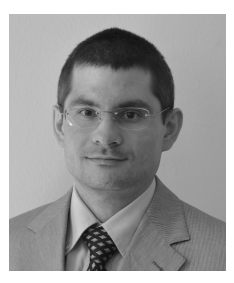

Markus A. Vogelsberger received his M.S. (with honors) and his Ph.D. (with honors) in Electrical Engineering from the Vienna University of Technology, Vienna, Austria, in 2004 and 2009, respectively. He has been a Scientific Research Assistant in the Institute of Electrical Drives and Machines, Vienna University of Technology, since he started working toward his Ph.D. His fields of interest include the transient excitations of induction machines, neural-network applications, optimized pulse width modulation and power electronics with an emphasis on three-phase drive converters. 


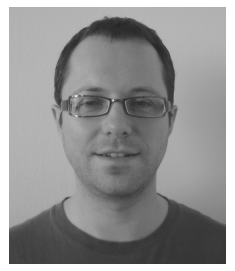

Peter E. Macheiner received his M.S. in Control Engineering in 2005 from the Vienna University of Technology. From 2005 to March 2009 he was a Project Assistant in the Department of Electrical Drives and Machines, Vienna University of Technology. He is currently a Research and Development Engineer with the Elin EBG Traction company. His fields of interest include condition monitoring for ac drives as well as control and simulation of electrical machines.

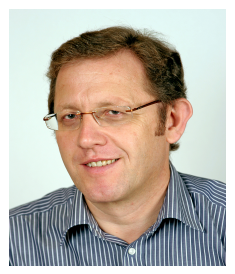

Pavol Bauer received his M.S. in Electrical Engineering at the Technical University of Kosice in 1985, his Ph.D. from the Delft University of Technology in 1995 and title of Professor from the president of the Czech Republic at the Brno University of Technology in 2008. Since 1990 he has been with the Delft University of Technology, teaching Power Electronics and Electrical Drives. From 2002 to 2003 he worked at KEMA (Arnhem) on different projects related to power electronics applications in power systems. Dr. Bauer has been published in over 42 journal and 190 conference papers in his field. He is also the author or co-author of 5 books, he holds an international patent and has organized several tutorials at international conferences. He has worked on many projects in industry concerning wind power and power electronic applications for power systems such as Smarttrafo etc. He has also participated in several Leonardo da Vinci EU projects as a project partner (ELINA, INETELE) and coordinator (PEMCWebLab.com). He is a Senior Member of the IEEE and a Chairman of the Benelux IEEE Joint Industry Applications Society and the Power Electronics and Power Engineering Society. He is also a member of the EPE-PEMC council, an EPE member and a member of international steering committees at numerous conferences.

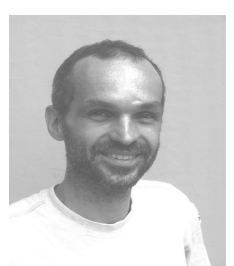

Thomas M. Wolbank studied Industrial Electronics at the Vienna University of Technology in Austria where he received his Ph.D. in 1996 and his Associate Professor Degree in 2004. At present he is with the Department of Electrical Drives and Machines at the Vienna University of Technology. 